\title{
Correlation Between Postoperative Early Recurrence of Hepatocellular Carcinoma and Mesenchymal Circulating Tumor Cells in Peripheral Blood
}

\author{
Zhong Wang $^{1}$ - Lei Luo ${ }^{1}$ - Yuan Cheng ${ }^{1}$ Guolin $\mathrm{He}^{1}$ • Bangjian Peng ${ }^{1} \cdot \mathrm{Yi} \mathrm{Gao}^{1}$ • \\ Ze-sheng Jiang ${ }^{1} \cdot$ MingXin Pan ${ }^{1}$
}

Received: 9 September 2017 / Accepted: 26 October 2017 / Published online: 20 November 2017

(C) 2017 The Author(s). This article is an open access publication

\begin{abstract}
Background Circulating tumor cells (CTCs) have been actively studied for their functions in hepatocellular carcinoma (HCC) recurrence. However, the relationship between circulating tumor cells subtypes and hepatocellular carcinoma recurrence is still unclear.

Methods CTCs were collected from the peripheral blood of 62 postoperative HCC patients. The CTCs were isolated with a filtration-based method. Multiplex fluorescence in situ hybridization was used to characterize the CTCs based on mRNA expression levels of epithelial and mesenchymal markers.

Results Of the 62 HCC patients, 26 were diagnosed with early recurrence (ER) and 36 did not experience recurrence. Comparison between the recurrence group and the non-recurrence group showed the total number of CTCs, mesenchymal CTCs, and mixed CTCs in the recurrence group was significantly higher than in the non-recurrence group. Receiver operator characteristic (ROC) curve analysis was performed to define the positive cutoff values as follows: total number of CTCs $\geq 4$, mesenchymal CTCs $\geq 1$, and mixed CTCs $\geq 3$. Analysis showed that portal vein tumor thrombus (hazard ratio $[\mathrm{HR}]=2.905$, $P=0.023)$ and mesenchymal CTC positivity ( $\mathrm{HR}=3.453, P=0.007)$ were independent risk factors for $\mathrm{ER}$. The correlation between the presence of mesenchymal CTCs and time to recurrence was further examined, and the results showed significantly shortened postoperative disease-free survival in patients positive for mesenchymal CTCs $(P<0.001)$.

Conclusions HCC patients with positive peripheral mesenchymal CTCs have a more serious risk of ER, which could be a potential biomarker in HCC prognosis monitoring.
\end{abstract}

Keywords Hepatocellular carcinoma (HCC) .

Epithelial-mesenchymal transition $($ EMT) $\cdot$ Circulating tumor cells (CTCs) · Early recurrence(ER)

\section{Introduction}

Hepatocellular carcinoma (HCC) is one of the most common malignancies in the world and has high morbidity and mortality. In recent years, the incidence of HCC has continued to

MingXin Pan

Pmxwxy@sohu.com

1 Second Department of Hepatobiliary Surgery, Zhujiang Hospital, Southern Medical University, Guangzhou 510282, China rise, especially in developing countries. Furthermore, the prognosis of HCC patients is still not optimistic, with a 5year survival rate of less than $20 \%$. Nowadays, surgical resection is the preferred treatment for most HCC patients. However, postoperative recurrence is the main factor that affects HCC prognosis. ${ }^{1,2}$ Currently, monitoring HCC recurrence mainly relies on ultrasonography, computed tomography (CT) examination, and serum alpha-fetoprotein (AFP) levels. ${ }^{3}$ However, these conventional monitoring methods cannot detect early recurrence in most cases. Therefore, it is crucial to identify sensitive biomarkers for the early diagnosis of $\mathrm{HCC}$ recurrence, which will facilitate the early detection of $\mathrm{HCC}$ recurrence and timely interventions to improve survival.

In 1896, Ashworth, an Australian researcher, found a type of cells that was very similar to tumor cells in the blood of a patient with a metastatic tumor, and he proposed the concept of circulating tumor cells (CTCs). ${ }^{4}$ Tumor recurrence and 
metastasis are very complex processes, which are usually considered to require CTC migration from the primary tumor focus into the peripheral blood. ${ }^{5}$ In addition, because peripheral CTC detection is a simple, reproducible, and minimally invasive procedure, CTCs have been actively studied over the last few decades for their functions in tumor diagnosis, recurrence, and metastasis. ${ }^{6-9}$ However, studies on the relationship between CTC subtypes and tumor recurrence have rarely been reported. In this study, peripheral CTC subtypes were examined in patients with HCC, and their relationship with HCC recurrence was analyzed. The clinical value of detecting each subtype of CTCs for early prediction of HCC recurrence was also investigated.

\section{Materials and Methods}

\section{Patient Selection}

Sixty-two hepatocellular carcinoma patients (58 males and 4 females 30-79 years old, with a median age of 55) who underwent radical resection at Zhujiang Hospital of Southern Medical University from March 2014 to March 2016 were enrolled in this prospective study. The inclusion criteria were as follows: (1) patients who underwent pathological specimen examination and had a definite pathological diagnosis of liver cancer according to the criteria set by the World Health Organization; (2) patients who underwent radical resection by an experienced physician, with no residual lesions at the margins of the excision site as confirmed via postoperative pathology examination; (3) patients who had not been treated with other anti-tumor therapies before the radical resection; and (4) patients who had no extrahepatic metastasis confirmed by preoperative imaging. Tumor stage was determined according to the Barcelona Clinic Liver Cancer (BCLC) staging classification, and the degree of tumor differentiation was defined according to the Edmondson-Steiner grading system.

\section{Follow-Up and Recurrence}

The patients entered the clinical follow-up period to monitor for recurrence after their peripheral blood samples were collected postoperatively (3-35 days after the surgery, with a median of 18 days). The patients underwent various followup examinations and treatments after surgery according to routine clinical schedules. Recurrence was defined as intrahepatic recurrence and extrahepatic metastasis from a comprehensive diagnosis based on imaging results from computed tomography (CT), magnetic resonance imaging (MRI), and digital subtraction angiography (DSA), or positron emission tomography (PET)-CT and serum AFP level and other examinations, with or without pathological diagnosis. Evidence of recurrence was considered as the end point.
Time to recurrence (TTR) was defined as the time interval between resection and the diagnosis of recurrence. Recurrence within 6 months after the surgery was defined as ER. Patients with no recurrence were followed up to September 30, 2016.

\section{Detection and Characterization of CTCs Using the CanPatrol ${ }^{\mathrm{TM}}$ System}

Five milliliters of peripheral blood sample from each patient was placed in a K2-ethylenediaminetetraacetic acid (EDTA) tube and centrifuged to collect cell pellets. The supernatant was discarded, and $5 \mathrm{~mL}$ of phosphate-buffered saline (PBS) was added to the tube to resuspend the cell pellet. The cell suspension was filtered through a filter tube (Surexam Biotech, Guangzhou, China) containing a membrane filter (Millipore, Billerica, MA, USA) with a pore size of $8 \mu \mathrm{m}$. The cell suspension was passed through the filter under vacuum, and the circulating tumor cells (CTCs) remained on the filter. As the diameter of blood cells is smaller than the diameter of CTCs, the blood cells passed through the filter. ${ }^{10}$

We designed three sets of nucleic acid probes to detect and characterize the expression levels of epithelial and mesenchymal genes in CTCs through multiplex RNA in situ hybridization (RNA-ISH). The first set of probes contained four epithelial transcripts (CK8, 18, and 19; EpCAM). The second set of probes consisted of two mesenchymal transcripts (Vimentin and Twist). The last set contained only CD45 transcripts to distinguish between leukocytes and CTCs. Detailed procedures for the hybridization followed previously published methods. ${ }^{11}$ Briefly, the cells remaining on the filter were permeabilized and digested with protease followed by a series of hybridization steps using the probes described above. Finally, the nuclei were stained with 4', 6-diamidino-2phenylindole (DAPI). The cells were analyzed using fluorescence microscopy. The red and green fluorescent signals observed in the cells represented the expression of the epithelial and mesenchymal genes, respectively. The blue fluorescent dots indicated CD45 expression, which is a marker of leukocytes. ${ }^{10,12}$

\section{Statistical Analysis}

The data were statistically analyzed using SPSS 21.0 statistical software. The CTCs and their subtypes were compared between patients with and without recurrence. The measurement data were analyzed using the rank-sum test (the measurement data did not exhibit a normal distribution on the normality test). The results for the predicted rates of recurrence based on different types of CTCs were used to plot receiver operator characteristic (ROC) curves, and the area under the curve (AUC) was calculated. The cutoff point corresponding to the maximum of the Youden index was taken as the best critical 
point for clinical diagnosis. The Kaplan-Meier test was used to compare the difference in survival rates between groups. The Cox proportional hazards regression model was used to compare the difference in survival rate. All the tests were bilateral, and the significance level was set at $\alpha=0.05$.

\section{Results}

\section{Relationship Between CTC Subtype and ER after Radical Resection}

To study the relationship between CTC subtypes and ER after radical resection, $62 \mathrm{HCC}$ patients, including 26 patients with ER (aged 30-74 years old with a median age of 52) and 36 patients without recurrence (aged 30-79 years old with a median age of 57.5), were followed up after surgery. Comparison between the recurrence group and the non-recurrence group showed a median of 6 vs 2.5 total CTCs, with 0.5 vs 0 median epithelial CTCs, 3 vs 1 median mixed CTCs, and 1 vs 0 median mesenchymal CTCs. A rank-sum test showed significantly more total CTCs $(P=0.011)$, mesenchymal CTCs $(P<0.001)$, and mixed CTCs $(P=0.027)$ in the peripheral blood of the recurrence group than in the non-recurrence group after HCC radical resection. No difference was observed in the epithelial CTC counts between the two groups $(P=0.619)($ Table 1$)$.

\section{Analysis of Risk Factors for Postoperative HCC Recurrence}

The cutoff values for each CTC subtype which was associated with ER were determined via ROC curve analysis, and the cutoff was considered positive for total CTCs $\geq 4$, mesenchymal CTCs $\geq 1$, and mixed CTCs $\geq 3$ (Fig. 1, Table 2).

To systematically analyze the relationship between ER after HCC radical resection and total CTCs, mesenchymal CTCs, mixed CTCs, or other factors, univariate Cox regression analysis was first performed for recurrence and each factor. The results showed a significant correlation between tumor recurrence and mesenchymal CTCs $(P<0.001)$, mixed CTCs $(P=0.009)$, total CTCs $(P=0.02)$, portal vein tumor thrombus $(P=0.001)$, and tumor size $(P=0.049)$. Multivariate Cox regression analysis further showed that mesenchymal CTCs (hazard ratio $[\mathrm{HR}]=3.453, P=0.007$ ) and portal vein tumor thrombus $(\mathrm{HR}=2.905, P=0.023)$ were independent risk factors for ER after HCC radical resection (Table 3), suggesting that mesenchymal CTCs have more predictive strength than portal vein tumor thrombus with respect to recurrence outcome. The correlation between mesenchymal CTCs and TTR was examined using the K-M test. Among the patients who were negative for portal vein tumor thrombus, the probability of recurrence was significantly higher in mesenchymal CTC-positive patients than in mesenchymal CTC-negative patients $(P<0.001$, Fig. 2$)$ over increasing follow-up durations.

\section{Discussion}

Currently, a large number of studies have demonstrated that epithelial-mesenchymal transition (EMT) plays a key role in tumor recurrence and metastasis. ${ }^{13,14}$ According to the EMT process, CTCs can be divided into different subtypes, including epithelial CTCs, mesenchymal CTCs, and mixed (epithelial/mesenchymal) CTCs. ${ }^{15}$ However, many techniques, including the CellSearch system, detect and isolate CTCs based only on epithelial markers, which is most likely to overlook the subpopulations of CTCs with undergoing EMT. ${ }^{16,17}$ An increasing number of studies have shown that tumor cells expressing mesenchymal markers lead to a poor prognosis for many tumors. ${ }^{18,19}$ To our knowledge, there is a lack of studies on the correlation between mesenchymal CTCs and postoperative HCC recurrence. Therefore, mesenchymal CTCs may be an ideal biomarker for predicting recurrence after radical resection of HCC. Moreover, the detection of CTCs can be used as a method for early intervention of HCC after radical resection. Thus, we used the secondgeneration CanPatrol ${ }^{\mathrm{TM}}$ CTC detection technology to isolate, identify, and classify CTCs in HCC patients. This technology can be used to classify CTCs in the peripheral blood into three categories based on EMT phenotype and to study the correlations between different CTC subtypes and ER of HCC. ${ }^{20}$

The present study examined the correlation between CTC phenotypes and early postoperative $\mathrm{HCC}$ recurrence, which was also the first study on the correlation between mesenchymal CTCs and HCC prognosis. It was demonstrated that total CTCs $(P=0.011)$, mesenchymal CTCs $(P<0.001)$, and mixed CTCs $(P=0.027)$ were positively correlated with
Table 1 The comparison of each CTC subtype count between recurrence and non-recurrence (median and interquartile range)

\begin{tabular}{lllll}
\hline & Total CTCs & Epithelial CTCs & Mixed CTCs & Mesenchymal CTCs \\
\hline Non-recurrence $(n=36)$ & $2.50(1.00-8.00)$ & $0.00(0.00-2.75)$ & $1.00(0.25-5.00)$ & $0.00(0.00-0.00)$ \\
Recurrence $(n=26)$ & $6.00(3.75-17.25)$ & $0.50(0.00-2.00)$ & $3.00(1.75-9.00)$ & $1.00(0.00-4.00)$ \\
$Z$ & 2.556 & 0.498 & 2.213 & 3.994 \\
$P$ value & 0.011 & 0.619 & 0.027 & 0.000 \\
\hline
\end{tabular}

$P<0.05$ was considered statistically significant 


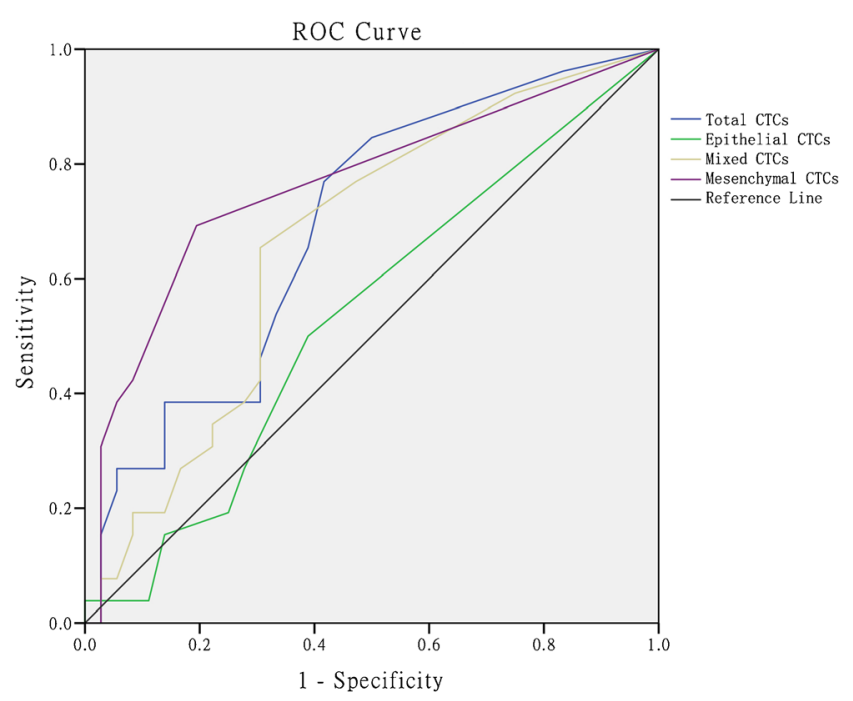

Fig. 1 ROC curves of different circulating tumor cell subtypes

postoperative recurrence through a rank-sum test. We found that the cutoff values for each CTC subtype to be positively correlated with recurrence through ROC curve analysis, with a definition of positive values for each CTC subtype (CTCs $\geq 4$, mesenchymal CTCs $\geq 1$, mixed CTCs $\geq 3$ ). Cox regression analysis showed that the risk of ER was significantly higher in mesenchymal CTC-positive patients than in mesenchymal CTC-negative patients ( $\mathrm{HR}=3.453, P=0.007)$. Meanwhile, a $\mathrm{K}-\mathrm{M}$ test showed significantly shortened postoperative disease-free survival in mesenchymal CTC-positive patients $(P<0.001)$.

The mechanisms underlying the formation of recurrent lesions by CTCs have been shown to be closely related to EMT and mesenchymal-epithelial transition (MET). ${ }^{18,21}$ Tumor cells generate highly invasive mesenchymal CTCs in the peripheral blood through the EMT process. ${ }^{22}$ Although the majority of mesenchymal CTCs are cleared by the immune system, a small number of mesenchymal CTCs escape from immune surveillance and remain in a dormant state. These mesenchymal CTCs undergo the MET process following changes in the body's immune activity and upon encountering specific microenvironments, resulting in recurrent colonization foci. ${ }^{23,24}$ According to the CTC formation hypothesis, tumor cells are spontaneously released into the peripheral blood during diagnostic or therapeutic procedures, leading to distant metastasis or intrahepatic recurrence when they return to the residual liver tissue. ${ }^{25}$ Before surgery, the patients were in a relatively stable state, which also meant their immune system was intact. Under such a condition, the internal environment was not considered suitable for CTC migration and colonization. However, due to the squeezing stimulation to the primary tumor foci during the operation, the tumor-neighboring microenvironment was damaged, which could lead to the shedding of a large number of tumor cells into the blood. ${ }^{26}$ In addition, postoperative internal environment imbalance could induce weakened ability of CTC clearance, which facilitates the colonization of highly invasive mesenchymal CTCs followed by tumor recurrence and metastasis. ${ }^{24}$ That is to say, patients who are preoperatively negative for CTCs may become postoperatively positive for CTCs due to surgical stimulation. Furthermore, the body is in a traditional "tumor-free" state after radical tumor resection. Accordingly, postoperative assessment of the relationship between CTCs and the recurrence of HCC not only allows relatively accurate predictions regarding recurrence but also excludes the impact of solid tumors per se on recurrence. Therefore, it is suggested that postoperative examination of the peripheral blood mesenchymal CTCs is more meaningful. Moreover, in prior studies of the relationship between CTCs in postoperative peripheral blood and tumor recurrence, CTCs were commonly collected within 4 5 weeks after surgery. ${ }^{27,28}$ Accordingly, we collected peripheral blood from HCC patients within 5 weeks after surgery to analyze the relationships between various CTC phenotypes and early recurrence. Our results further confirmed that postoperative mesenchymal CTCs were positively correlated with $\mathrm{HCC}$ recurrence and that the disease-free survival was significantly shortened in mesenchymal CTC-positive patients compared to mesenchymal CTC-negative patients.

Present results suggest that mesenchymal CTCs are not only associated with HCC recurrence but is also helpful in the prediction of early recurrence. Therefore, examination of peripheral mesenchymal CTCs in postoperative HCC patients
Table 2 Diagnostic values of CTC count at selected cutoff point

\begin{tabular}{llllll}
\hline CTC subtype & Cutoff point & Sensitivity & Specificity & YI & AUC \\
\hline Total CTCs & $\geq 3$ & 0.846 & 0.500 & 0.346 & 0.691 \\
& $\geq 4$ & 0.769 & 0.583 & 0.352 & \\
Mixed CTCs & $\geq 5$ & 0.654 & 0.611 & 0.265 & \\
& $\geq 2$ & 0.769 & 0.528 & 0.297 & 0.664 \\
Mesenchymal CTCs & $\geq 3$ & 0.654 & 0.694 & 0.348 & \\
& $\geq 4$ & 0.423 & 0.694 & 0.117 & \\
& $\geq 2$ & 0.692 & 0.806 & 0.498 & 0.764 \\
& $\geq 3$ & 0.423 & 0.917 & 0.340 & \\
\hline
\end{tabular}


Table 3 Analyses of risk factors for HCC early recurrence

\begin{tabular}{|c|c|c|c|c|c|c|c|c|c|}
\hline & & \multicolumn{2}{|c|}{ Recurrence } & \multicolumn{3}{|l|}{ Univariate analysis } & \multicolumn{3}{|l|}{ Multivariate analysis } \\
\hline & & No & Yes & $\operatorname{HR}(95 \% \mathrm{CI})$ & $\chi^{2}$ & $P^{*}$ & $\mathrm{HR}(95 \% \mathrm{CI})$ & $\chi^{2}$ & $P$ \\
\hline Age (years) & $\begin{array}{l}\leq 50 \\
>50\end{array}$ & $\begin{array}{l}8 \\
28\end{array}$ & $\begin{array}{l}10 \\
16\end{array}$ & $0.586(0.266-1.291)$ & 1.759 & 0.185 & - & - & - \\
\hline Gender & $\begin{array}{l}\text { Male } \\
\text { Female }\end{array}$ & $\begin{array}{l}33 \\
3\end{array}$ & $\begin{array}{l}25 \\
1\end{array}$ & $0.501(0.068-3.695)$ & 0.460 & 0.497 & - & - & - \\
\hline Tumor size (cm) & $\begin{array}{l}\leq 5 \mathrm{~cm} \\
>5 \mathrm{~cm}\end{array}$ & $\begin{array}{l}18 \\
18\end{array}$ & $\begin{array}{l}7 \\
19\end{array}$ & $2.393(1.003-5.710)$ & 3.867 & 0.049 & NS & & \\
\hline Cirrhosis & $\begin{array}{l}\text { No } \\
\text { Yes }\end{array}$ & $\begin{array}{l}25 \\
11\end{array}$ & $\begin{array}{l}17 \\
9\end{array}$ & $1.203(0.536-2.700)$ & 0.201 & 0.654 & - & - & - \\
\hline $\begin{array}{l}\text { Portal vein } \\
\text { Tumor thrombus }\end{array}$ & $\begin{array}{l}\text { No } \\
\text { Yes }\end{array}$ & $\begin{array}{l}34 \\
2\end{array}$ & $\begin{array}{l}17 \\
9\end{array}$ & $4.275(1.852-9.869)$ & 11.585 & 0.001 & $2.905(1.159-7.280)$ & 5.179 & 0.023 \\
\hline Child-Pugh class & $\begin{array}{l}\mathrm{A} \\
\mathrm{B}\end{array}$ & $\begin{array}{l}32 \\
4\end{array}$ & $\begin{array}{l}20 \\
6\end{array}$ & $1.842(0.737-4.606)$ & 1.708 & 0.191 & - & - & - \\
\hline HBsAg & $\begin{array}{l}\text { Negative } \\
\text { Positive }\end{array}$ & $\begin{array}{l}6 \\
30\end{array}$ & $\begin{array}{l}5 \\
21\end{array}$ & $0.944(0.356-2.504)$ & 0.014 & 0.907 & - & - & - \\
\hline $\operatorname{AFP}(\mu \mathrm{g} / \mathrm{L})$ & $\begin{array}{l}\leq 400 \\
>400\end{array}$ & $\begin{array}{l}28 \\
8\end{array}$ & $\begin{array}{l}15 \\
11\end{array}$ & $2.181(0.996-4.776)$ & 3.798 & 0.051 & NS & & \\
\hline CEA $(\mu \mathrm{g} / \mathrm{L})$ & $\begin{array}{l}\leq 5 \\
>5\end{array}$ & $\begin{array}{l}32 \\
4\end{array}$ & $\begin{array}{l}22 \\
4\end{array}$ & $1.311(0.451-3.808)$ & 0.247 & 0.619 & - & - & - \\
\hline CA199 (kU/L) & $\begin{array}{l}\leq 34 \\
>34\end{array}$ & $\begin{array}{l}27 \\
9\end{array}$ & $\begin{array}{l}16 \\
10\end{array}$ & $1.699(0.770-3.751)$ & 1.720 & 0.190 & - & - & - \\
\hline Total CTCs & $\begin{array}{l}\text { Negative } \\
\text { Positive }\end{array}$ & $\begin{array}{l}21 \\
15\end{array}$ & $\begin{array}{l}6 \\
20\end{array}$ & $2.950(1.184-7.349)$ & 5.393 & 0.020 & NS & & \\
\hline Mixed CTCs & $\begin{array}{l}\text { Negative } \\
\text { Positive }\end{array}$ & $\begin{array}{l}25 \\
11\end{array}$ & $\begin{array}{l}9 \\
17\end{array}$ & $2.935(1.306-6.594)$ & 6.797 & 0.009 & NS & & \\
\hline Mesenchymal CTCs & $\begin{array}{l}\text { Negative } \\
\text { Positive }\end{array}$ & $\begin{array}{l}29 \\
7\end{array}$ & $\begin{array}{l}8 \\
18\end{array}$ & $4.740(2.041-11.010)$ & 13.099 & 0.000 & $3.453(1.393-8.559)$ & 7.157 & 0.007 \\
\hline Edmondson stage & $\begin{array}{l}\text { I-II } \\
\text { III-IV }\end{array}$ & $\begin{array}{l}35 \\
1\end{array}$ & $\begin{array}{l}23 \\
3\end{array}$ & $0.343(0.103-1.148)$ & 3.011 & 0.083 & NS & & \\
\hline BCLC stage & $\begin{array}{l}0+A \\
B+C\end{array}$ & $\begin{array}{l}14 \\
22\end{array}$ & $\begin{array}{l}9 \\
17\end{array}$ & $1.362(0.606-3.060)$ & 0.560 & 0.454 & - & - & - \\
\hline ALP (U/L) & $\begin{array}{l}\leq 160 \\
>160\end{array}$ & $\begin{array}{l}32 \\
4\end{array}$ & $\begin{array}{l}19 \\
7\end{array}$ & $2.178(0.906-5.236)$ & 3.025 & 0.082 & NS & & \\
\hline ALT (U/L) & $\begin{array}{l}\leq 75 \\
>75\end{array}$ & $\begin{array}{l}27 \\
9\end{array}$ & $\begin{array}{l}22 \\
4\end{array}$ & $0.656(0.225-1.907)$ & 0.601 & 0.438 & - & - & - \\
\hline
\end{tabular}

$* P<0.10$ will be further multivariate analysis

NS non-significance, AFP alpha-fetoprotein, HBsAg hepatitis B surface antigen, CEA carcinoembryonic antigen, CA199 carbohydrate antigen 199 , $B C L C$ stage Barcelona Clinic Liver Cancer stage, $A L P$ alkaline phosphatase, $A L T$, alanine aminotransferase

can be a good secondary indicator in monitoring the prognosis of HCC patients, especially when traditional monitoring methods cannot provide evidence for the diagnosis. This method could improve the early diagnosis of postoperative HCC recurrence, facilitate early personalized interventions in postoperative patients with positive results for mesenchymal CTCs, and prolong their survival. ${ }^{21,29}$ In addition, CTCs of different subtypes have different properties and show different responses to the same treatment. ${ }^{30}$ So, for HCC patients who are postoperatively positive for mesenchymal CTCs, we can eliminate mesenchymal CTCs in the peripheral blood via chemotherapy and other treatments to effectively block the formation of recurrence after surgery and thereby achieve the early prevention of HCC recurrence. ${ }^{31}$ This finding may provide new ideas for the development of new medicines that act on mesenchymal CTCs. Therefore, in future studies, we will also focus on drugs that can effectively reduce CTCs in the peripheral blood of patients with HCC to prevent early recurrence.

As described above, CTCs detected after radical resection of a tumor might be derived from two sources: (1) CTCs that were preoperatively present in peripheral blood and (2) CTCs produced when tumor cells were torn from tumor foci and released into the circulating blood due to mechanical stimulation of these foci and changes in the environment of the body during surgery. And the formation of recurrent lesions is 


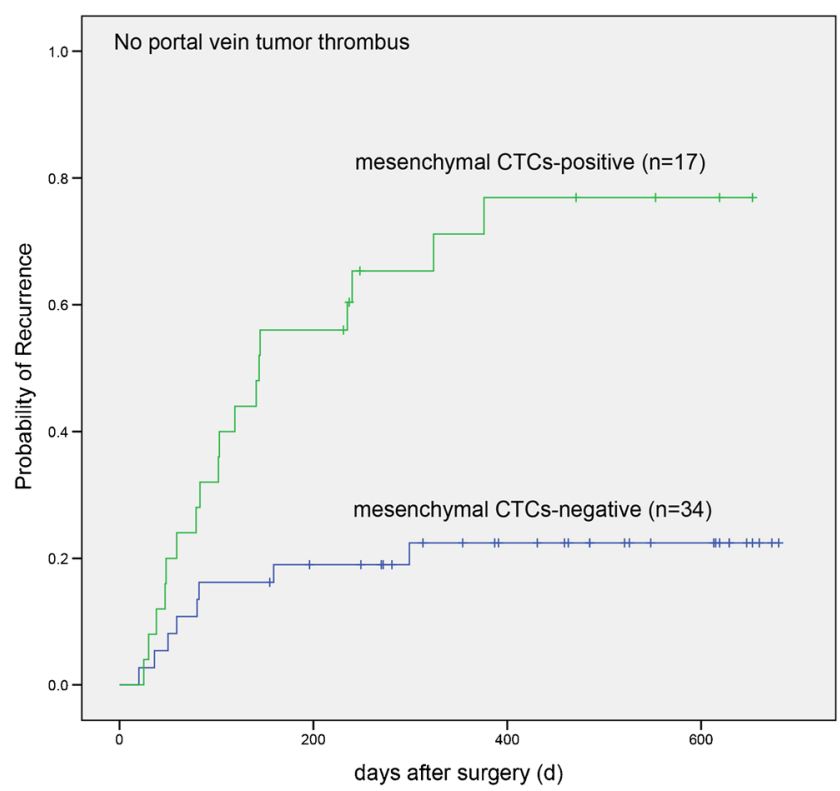

Fig. 2 HCC patients without portal vein tumor thrombus: log-rank test for time to recurrence with mesenchymal CTC-positive or negative postoperatively

related to both the body's immune status and the invasive ability of CTCs per se. In conjunction with our study results, it indicates that mesenchymal CTCs may largely arise from a CTC subtype with strong invasion and colonization capabilities. Therefore, we will further study the functional characteristics and properties of mesenchymal CTCs in HCC to elucidate the underlying role of mesenchymal CTCs in the molecular mechanism of recurrent foci formation in HCC. On the basis of this study, we will conduct a prospective, multicenter clinical study to further validate the relationship between mesenchymal CTCs and HCC prognosis and further analyze the effects of surgical procedure on CTC phenotypes and quantities to optimize the surgical procedure.

\section{Conclusions}

In conclusion, we found that postoperative total CTCs, mixed CTCs, and mesenchymal CTCs count was significantly higher in recurrence patients of hepatocellular carcinoma than in nonrecurrence patients, and there is no difference in postoperative epithelial CTC counts between the two groups. Furthermore, we demonstrated that postoperative mesenchymal CTCpositive in the peripheral blood indicates early recurrence of hepatocellular carcinoma after curative resection. Monitoring postoperative mesenchymal CTCs may be a promising predictor of early recurrence of $\mathrm{HCC}$, and eradicating these cells might open a therapeutic avenue toward preventing $\mathrm{HCC}$ recurrence.
Acknowledgements We would like to thank all the patients who participated in this study and the nurses who collected the specimens at Zhujiang Hospital as well as Surexam Biotech (Guangzhou, China) for their technical support.

Authors' Contributions ZW analyzed the data and wrote the manuscript. ZW, LL, YC, and GH performed the experiments. LL and BP collected the patients' information. YG, ZJ, and MXP designed the study, edited the manuscript, and confirmed the data presented in the manuscript. All authors read and approved the final manuscript.

Funding This work was supported by grants from Natural Science Foundation of Guangdong Province, China, No.2016A030313626. The funding had no role in the collection, analysis, and interpretation of data, design of the study, or writing of the manuscript.

\section{Compliance with Ethical Standards}

Ethics Approval and Consent to Participate This study was approved by the Clinical Research Ethics Committee of Zhujiang Hospital of Southern Medical University (with numbers ZJYY-2014-GDEK-002). All participants provided informed written consent prior to their entry into the study. In the case that changes in the protocol are necessary, relevant amendments are made and submitted to the ethics trial registration authorities for approval.

Competing Interests The authors declare that they have no competing interests.

Availability of Data and Materials Please contact the author for data requests.

Open Access This article is distributed under the terms of the Creative Commons Attribution 4.0 International License (http:// creativecommons.org/licenses/by/4.0/), which permits unrestricted use, distribution, and reproduction in any medium, provided you give appropriate credit to the original author(s) and the source, provide a link to the Creative Commons license, and indicate if changes were made.

\section{References}

1. Shah SA, Greig PD, Gallinger S, Cattral MS, Dixon E, Kim RD, Taylor BR, Grant DR, Vollmer CM. Factors associated with early recurrence after resection for hepatocellular carcinoma and outcomes. J Am Coll Surg 2006; 202:275-283.

2. Wang R, Chen XZ, Zhang MG, Tang L, Wu H. Incidence and mortality of liver cancer in mainland China: changes in first decade of 21st century. Hepato-Gastroenterology 2015; 62:118-121.

3. Colombo F, Baldan F, Mazzucchelli S, Martin-Padura I, Marighetti P, Cattaneo A, Foglieni B, Spreafico M, Guerneri S, Baccarin M et al. Evidence of distinct tumour-propagating cell populations with different properties in primary human hepatocellular carcinoma. Plos One 2011; 6:e21369.

4. Pantel K, Alix-Panabieres C. Circulating tumour cells in cancer patients: challenges and perspectives. Trends Mol Med 2010; 16 : 398-406.

5. Imamura H, Matsuyama Y, Tanaka E, Ohkubo T, Hasegawa K, Miyagawa S, Sugawara Y, Minagawa M, Takayama T, Kawasaki $\mathrm{S}$ et al. Risk factors contributing to early and late phase intrahepatic recurrence of hepatocellular carcinoma after hepatectomy. J Hepatol 2003; 38:200-207. 
6. Kaifi JT, Kunkel M, Das A, Harouaka RA, Dicker DT, Li G et al. Circulating tumor cell isolation during resection of colorectal cancer lung and liver metastases: a prospective trial with different detection techniques. Cancer Biol Ther 2015;16:699-708.

7. Joosse SA, Gorges TM, Pantel K. Biology, detection, and clinical implications of circulating tumor cells. Embo Mol Med 2014; 7:1-11.

8. Aceto N, Bardia A, Miyamoto DT, Donaldson MC, Wittner BS, Spencer JA, Yu M, Pely A, Engstrom A, Zhu H et al. Circulating tumor cell clusters are oligoclonal precursors of breast cancer metastasis. Cell 2014; 158:1110-1122.

9. Cristofanilli M. Circulating tumour cells: telling the truth about metastasis. Lancet Oncol 2014; 15:365-366.

10. Liu Y, Hu B, Li Z, He X, Li Y, Lu L. An improved strategy to detect the epithelial-mesenchymal transition process in circulating tumor cells in hepatocellular carcinoma patients. Hepatol Int 2016; 10: 640-646.

11. Wu S, Liu Z, Liu S, Lin L, Yang W, Xu J. Enrichment and enumeration of circulating tumor cells by efficient depletion of leukocyte fractions. Clin Chem Lab Med 2014; 52:243-251.

12. Li TT, Liu H, Li FP, et al. Evaluation of epithelial-mesenchymal transitioned circulating tumor cells in patients with resectable gastric cancer: relevance to therapy response. World J Gastroenterol 2015; 21:13259-13267.

13. Barrière G, Tartary M, Rigaud M. Epithelial mesenchymal transition: a new insight into the detection of circulating tumor cells. Isrn Oncology 2012; 2012: 382010 .

14. Li Y, Xu S, Li J, Han K, Pi H, Zheng L, Zuo G, Huang X, Li H, Zhao $\mathrm{H}$ et al. Epithelial-mesenchymal transition markers expressed in circulating tumor cells in hepatocellular carcinoma patients with different stages of disease. Cell Death and Disease 2013; 4:e831.

15. Wu S, Liu S, Liu Z, Huang J, Pu X, Li J, Yang D, Deng H, Yang N, Xu J. Classification of circulating tumor cells by epithelial-mesenchymal transition markers. Plos One 2015;10: UNSP e0123976.

16. Harouaka R, Kang Z, Zheng SY, Cao L. Circulating tumor cells: advances in isolation and analysis, and challenges for clinical applications. Pharmacol Ther 2014; 141:209-221.

17. Hong B, $\mathrm{Zu} \mathrm{Y}$. Detecting circulating tumor cells: current challenges and new trends. Theranostics 2013; 3:377-394.

18. Yu M, Bardia A, Wittner BS, Stott SL, Smas ME, Ting DT, Isakoff SJ, Ciciliano JC, Wells MN, Shah AM et al. Circulating breast tumor cells exhibit dynamic changes in epithelial and mesenchymal composition. Science 2013; 339:580-584.

19. Si Y, Lan G, Deng Z, Wang Y, Lu Y, Qin Y, Huang B, Yang Y, Weng J, Han $X$ et al. Distribution and clinical significance of circulating tumor cells in nasopharyngeal carcinoma. Jpn J Clin Oncol 2016; 46:622-630.

20. Guan X, Ma F, Liu S, Wu S, Xiao R, Yuan L, Sun X, Yi Z, Yang H, $\mathrm{Xu} \mathrm{B}$. Analysis of the hormone receptor status of circulating tumor cell subpopulations based on epithelial-mesenchymal transition: a proof-of-principle study on the heterogeneity of circulating tumor cells. Oncotarget 2016; 7: 65993-66002.

21. Zhang Y, Shi ZL, Yang X, Yin ZF. Targeting of circulating hepatocellular carcinoma cells to prevent postoperative recurrence and metastasis. World J Gastroenterol 2014; 20: 142-147

22. Yoshida T, Ozawa Y, Kimura T, Sato Y, Kuznetsov G, Xu S, Uesugi M, Agoulnik S, Taylor N, Funahashi Y et al. Eribulin mesilate suppresses experimental metastasis of breast cancer cells by reversing phenotype from epithelial-mesenchymal transition (EMT) to mesenchymal-epithelial transition (MET) states. Br J Cancer 2014; 110:1497-1505.

23. Li YM, Xu SC, Li J, Han KQ, Pi HF, Zheng L, Zuo GH, Huang XB, Li HY, Zhao HZ et al. Epithelial-mesenchymal transition markers expressed in circulating tumor cells in hepatocellular carcinoma patients with different stages of disease. Cell Death Dis 2013; 4:e831.

24. Liu S, Li N, Yu X, Xiao X, Cheng K, Hu J, Wang J, Zhang D, Cheng S, Liu S. Expression of intercellular adhesion molecule 1 by hepatocellular carcinoma stem cells and circulating tumor cells. Gastroenterology 2013; 144:1031-1041.

25. Xu W, Cao L, Chen L, Li J, Zhang XF, Qian HH, Kang XY, Zhang Y, Liao J, Shi LH et al. Isolation of circulating tumor cells in patients with hepatocellular carcinoma using a novel cell separation strategy. Clin Cancer Res 2011; 17:3783-3793.

26. Sawabata N, Funaki S, Hyakutake T, Shintani Y, Fujiwara A, Okumura M. Perioperative circulating tumor cells in surgical patients with non-small cell lung cancer: does surgical manipulation dislodge cancer cells thus allowing them to pass into the peripheral blood? Surg Today 2016;46:1402-1409.

27. Hao S, Chen S, Tu C, Huang T. Anterior approach to improve the prognosis in HCC patients via decreasing dissemination of EpCAM+ circulating tumor cells. J Gastrointest Surg 2017;21: $1112-1120$.

28. Sun YF, Xu Y, Yang XR, et al. Circulating stem cell-like epithelial cell adhesion molecule-positive tumor cells indicate poor prognosis of hepatocellular carcinoma after curative resection. Hepatology 2013;57:1458-1468.

29. Franken B, de Groot MR, Mastboom WJ, Vermes I, van der Palen J, Tibbe AG, Terstappen LW. Circulating tumor cells, disease recurrence and survival in newly diagnosed breast cancer. Breast Cancer Res 2012; 14:R133.

30. Raimondi C, Gradilone A, Naso G, Vincenzi B, Petracca A, Nicolazzo C, Palazzo A, Saltarelli R, Spremberg F, Cortesi E et al. Epithelial-mesenchymal transition and stemness features in circulating tumor cells from breast cancer patients. Breast Cancer Res Treat 2011; 130:449-455.

31. Zhang $\mathrm{Y}$, Zhang $\mathrm{X}$, Zhang J, et al. Microfluidic chip for isolation of viable circulating tumor cells of hepatocellular carcinoma for their culture and drug sensitivity assay. Cancer Biol Ther 2016;17:1177-1187. 